\title{
Buy-Many Mechanisms are Not Much Better than Item Pricing
}

\author{
SHUCHI CHAWLA, YIFENG TENG, and CHRISTOS TZAMOS, University of Wisconsin-Madison
}

\author{
CCS Concepts: • Theory of computation $\rightarrow$ Algorithmic mechanism design; Computational pricing \\ and auctions.
}

Additional Key Words and Phrases: revenue maximization, lotteries, simple mechanisms, menu-size complexity

ACM Reference Format:

Shuchi Chawla, Yifeng Teng, and Christos Tzamos. 2019. Buy-Many Mechanisms are Not Much Better than Item Pricing. In ACM EC '19: ACM Conference on Economics and Computation (EC '19), fune 24-28, 2019, Phoenix, AZ, USA. ACM, New York, NY, USA, 2 pages. https://doi.org/10.1145/3328526.3329583

It is well known that multi-item revenue optimal mechanisms can be very complex. Expressed as menus where each option corresponds to an allocation paired with a price, optimal mechanisms may price many different bundles of items at different prices, and even offer an unbounded number of random allocations or lotteries [3]. Such complexity is thought to be necessary as the revenue gaps between randomized and deterministic mechanisms, or between deterministic and simple mechanisms, are huge even for simple classes of valuations. We challenge this conventional belief by showing that these large gaps can only happen in situations where buyers' actions are severely restricted. These are situations where the mechanism sells a bundle of items at a higher price than the sum of the prices of the constituent items and buyers wanting to purchase such a bundle must pay this premium as they are not allowed to purchase the constituents separately.

We consider Sybil-proof or buy-many mechanisms where the buyer is allowed to purchase any multi-set of menu options (of arbitrary size); The buyer pays the sum of the prices of the menu options and obtains the union of the corresponding allocations. ${ }^{1}$ Our main result is that the revenue obtained by a Sybil-proof or buy-many mechanism is at most a logarithmic factor larger than the revenue achievable using a particular class of simple mechanisms, namely item pricing. ${ }^{2}$ This holds in the most general setting possible, with an arbitrarily correlated distribution of buyer types and arbitrary valuations over $n$ items. Denoting by SybilProofRev the optimal revenue achievable through (potentially randomized) Sybil-proof mechanisms, and SREv the optimal revenue obtained by an item pricing, our main theorem is:

THeorem 1. For any distribution over arbitrary valuation functions, $\frac{\text { SybiLPRoofRev }}{\text { SRev }}=O(\log n)$.

\footnotetext{
${ }^{1}$ When the menu contains lotteries, each lottery bought by the buyer is instantiated independently. There is a slight distinction between whether the buyer can select a multi-set of options adaptively depending on outcomes of previous lotteries, or non-adaptively. Our results apply to both settings.

${ }^{2} \mathrm{An}$ item pricing is an additive pricing function: items are assigned prices and the cost of a set is the sum of its item prices.
}

Permission to make digital or hard copies of part or all of this work for personal or classroom use is granted without fee provided that copies are not made or distributed for profit or commercial advantage and that copies bear this notice and the full citation on the first page. Copyrights for third-party components of this work must be honored. For all other uses, contact the owner/author(s).

EC '19, June 24-28, 2019, Phoenix, AZ, USA

(C) 2019 Copyright held by the owner/author(s).

ACM ISBN 978-1-4503-6792-9/19/06.

https://doi.org/10.1145/3328526.3329583 
In contrast, previous work showed that for any $n>1$ there exist distributions over additive valuations for which REv/DREv $=\infty$ and DREv/SREv $=2^{\Omega(n)}$ [6], as well as distributions over unitdemand valuations for which Rev/SRev $=\infty$ [2]. Here Rev denotes the optimal revenue obtained by any randomized IC mechanism, and DREv the optimal revenue obtained by any deterministic mechanism. Our result implies that Sybil-proofness places a significant restriction on the seller-it can lower the seller's revenue by an unboundedly large factor.

We show that this result is tight in a very strong sense as even very complex mechanisms cannot get better than logarithmic approximation to SybilProofRev.

Theorem 2. No mechanism with sub-exponential description complexity can obtain a o $(\log n)$ fraction of the optimal deterministic Sybil-proof revenue, even for additive buyers.

Item pricing matches this bound against randomized mechanisms while having only linear description complexity. Our results directly extend the work of Briest et al. [2] who previously proved the same logarithmic upper and lower bounds for the special case of unit-demand buyers.

Implications for menu size complexity. The menu size of an auction, defined as the number of different outcomes the seller offers to the buyer, has been studied extensively in literature as a measure of complexity for single-buyer mechanisms (see, e.g., [1, 4-6]). Hart and Nisan [6] introduced the concept of "additive menu size", namely the number of "basic" options a buy-many mechanism offers, and showed that even mechanisms with small additive menu size cannot capture a good fraction of the optimal revenue. Our results show that allowing the buyer to purchase multiple options doesn't just allow a more succinct description of the mechanism, it also fundamentally changes the set of mechanisms available to the seller. Our work calls for a renewed investigation of additive menu-size complexity of Sybil-proof mechanisms.

Our techniques. Underlying our upper bound is the following general theorem that may be of independent interest. We show that if a pricing function $g$ point-wise c-approximates a pricing function $f$, then a particular (random) scaling of $g$ obtains an $O(\log c)$-approximation in revenue to $f$ with respect to any arbitrary distribution over valuation functions.

THEOREM 3. For any $c>1$, let $f$ and $g$ be any two pricing functions satisfying $g(\lambda) / c \leq f(\lambda) \leq$ $g(\lambda)$ for all random allocations $\lambda \in \Delta\left(2^{[n]}\right)$. Then there exists a distribution over "scaling factors" $\alpha \in[1 / 2 c, 1]$ such that for any valuation function $v, \mathrm{E}_{\alpha}\left[\operatorname{Rev}_{v}(\alpha g)\right] \geq \frac{1}{2 \log (2 c)} \operatorname{REv}_{v}(f)$.

Theorem 3 is easy to prove for single-minded buyers, but for general valuations requires a careful handling of incentives. Obtaining Theorem 1 then merely requires showing that additive functions approximate Sybil-proof pricings pointwise within a factor of $n$. This is straightforward to show for adaptively Sybil-proof menus. Non-adaptively Sybil-proof menus require an additional argument.

Acknowledgements. We thank the reviewers for suggesting the specific form of Theorem 3.

Full paper. https://arxiv.org/pdf/1902.10315.pdf

\section{REFERENCES}

[1] Moshe Babaioff, Yannai A. Gonczarowski, and Noam Nisan. 2017. The Menu-size Complexity of Revenue Approximation. In Proceedings of the 49th Annual ACM SIGACT Symposium on Theory of Computing (STOC 2017). 869-877.

[2] Patrick Briest, Shuchi Chawla, Robert Kleinberg, and S. Matthew Weinberg. 2010. Pricing Randomized Allocations. In ACM Symp. on Discrete Algorithms. 585-597.

[3] Constantinos Daskalakis, Alan Deckelbaum, and Christos Tzamos. 2013. Mechanism design via optimal transport. In ACM Conf. on Electronic Commerce. 269-286.

[4] Shaddin Dughmi, Li Han, and Noam Nisan. 2014. Sampling and Representation Complexity of Revenue Maximization. In Web and Internet Economics. Springer International Publishing, 277-291.

[5] Yannai A. Gonczarowski. 2018. Bounding the Menu-size of Approximately Optimal Auctions via Optimal-transport Duality. In Proceedings of the 50th Annual ACM SIGACT Symposium on Theory of Computing (STOC 2018). 123-131.

[6] Sergiu Hart, Noam Nisan, et al. 2013. The menu-size complexity of auctions. Center for the Study of Rationality. 